\title{
Superconductivity near Itinerant Ferromagnetic Quantum Criticality
}

\author{
Ziqiang $\mathrm{Wang}^{a}$, Wenjin $\mathrm{Mao}^{a, b}$, and Kevin Bedell ${ }^{a}$ \\ ${ }^{a}$ Department of Physics, Boston College, Chestnut Hill, MA 02467 \\ ${ }^{b}$ Department of Physics, Rutgers University, Piscataway, NJ 08854
}

(November 12, 2018)

\begin{abstract}
Superconductivity mediated by spin fluctuations in weak and nearly ferromagnetic metals is studied close to the zero-temperature magnetic transition. We solve analytically the Eliashberg equations for $p$-wave pairing and obtain the normal state quasiparticle self-energy and the superconducting transition temperature $T_{c}$ as a function of the distance to the quantum critical point. We show that the reduction of quasiparticle coherence and life-time due to scattering by quasistatic spin fluctuations is the dominant pair-breaking process, which leads to a rapid suppression of $T_{c}$ to a nonzero value near the quantum critical point. We point out the differences and the similarities of the problem to that of the theory of superconductivity in the presence of paramagnetic impurities.
\end{abstract}

PACS numbers: 74.20.Mn, 71.20.Lp, 74.72.Yg, 75.10.Lp

Superconducting ground states have recently been discovered in materials sitting close to the zero-temperature phase boundary of both antiferromagnetic and ferromagnetic transitions [1.2]. It is quite natural to suspect that paring in these materials is mediated by spin-fluctuations which are enhanced near the magnetic quantum critical point (QCP) [3 9]. However, it is known that quasiparticles scattered by critical spin-fluctuations are subject to severe non-Fermi liquid self-energy corrections that are in general pair-breaking. The interplay between these two competing effects, generic to superconductivity near quantum phase transitions, has not been fully understood and is the subject of this work.

We focus on the case of p-wave superconductivity near the ferromagnetic QCP where the Curie temperature is driven to zero by, e.g. pressure or doping [2]10]. In the vicinity of the transition, the metals can be described by weak ferromagnetic (FM) and nearly FM Fermi liquids at low temperatures, respectively [11,12]. The important low energy excitations are the quasiparticles and the long wavelength spin fluctuations. Since the quasiparticles couple strongly to spin fluctuations near the QCP, it is necessary to adapt the framework of the strong coupling Eliashberg equations. The long wavelength nature of the critical spin fluctuations near the ferromagnetic QCP [13] makes the solution of these equations theoretically tractable, enabling us to obtain analytical results for the self-energy and the transition temperature $T_{c}$. We find the onset of p-wave superconductivity in both the Fermi liquid $T_{c}<T^{*}$ and the quantum critical regime $T_{c}>T^{*}$, where $T^{*}$ is the characteristic frequency for spin fluctuations. We show that $T_{c}$ is rapidly reduced on approaching quantum criticality with $T^{*} \rightarrow 0$ due to the rapid reduction of quasiparticle coherence and lifetime caused by quasistatic scattering of spin fluctuations analogous to the suppression of $T_{c}$ by paramagnetic impurities [14]. Interestingly, $T_{c}$ remains finite at the FM QCP. We shall not discuss the problem of coexistence of ferromagnetism and superconductivity here, because the latter requires a careful treatment of the coupling between the superconducting order parameter and the electromagnetic fields associated with the ferromagnetic fluctuations 15 that is beyond the scope of this work.

In a FM Fermi liquid with spin polarization along the $z$-axis, the single-particle Green's function has the form, $G_{\sigma}(\epsilon, \vec{p})=a_{\sigma} /\left[\epsilon-v_{\sigma}\left(\vec{p}-p_{\sigma}\right)+i \eta \operatorname{sgn}(\epsilon)\right]$, where $\eta \rightarrow 0^{+}$, $p_{\sigma}(\sigma=\uparrow, \downarrow)$ are the Fermi momenta of the spin up and down electrons, and $a_{\sigma}$ are the wave-function renormalizations. For a weak FM metal, such as the one close to a continuous FM transition, the difference in the Fermi momenta is small, $\delta \equiv\left|p_{\uparrow}-p_{\downarrow}\right| \ll p_{\uparrow, \downarrow}$, and it is possible to set $p_{\uparrow}=p_{\downarrow}=p_{F}$ and $a_{\uparrow}=a_{\downarrow}$ [11]. The spin fluctuations are described by the propagators of the electron spindensity, $D_{i j}(\vec{x}, \tau)=-i\left\langle\left(S_{i}(\vec{x}, \tau)-\left\langle S_{i}\right\rangle\right)\left(S_{j}(0,0)-\left\langle S_{j}\right\rangle\right)\right\rangle$, $i, j=x, y, z$. The long wavelength and low energy spinfluctuations in the transverse and longitudinal channels are given by [11,

$$
\begin{aligned}
D_{\|}(\omega, \vec{q}) & =-\frac{N_{F}}{2} \frac{1}{\alpha+\left(\frac{q}{2 p_{F}}\right)^{2}-i \frac{\pi \omega}{4 \Lambda} \frac{2 p_{F}}{q}}, \\
D_{\perp}(\omega, \vec{q}) & = \begin{cases}-\frac{N_{F}}{2} \frac{1}{\left(\frac{q}{2 p_{F}}\right)^{2}-i \frac{\pi \omega}{4 \Lambda} \frac{2 p_{F}}{q}}, & q \gg|\delta|, \\
-S \frac{\omega_{s}(q)}{\omega^{2}-\omega_{s}^{2}(q)}, & q \ll|\delta| .\end{cases}
\end{aligned}
$$

Here $\alpha \approx\left(\delta / p_{F}\right)^{2} \ll 1$ measures the distance to the critical point, $S=v_{F} \delta N_{F} / 2$ is the averaged uniform spin density, $\Lambda=2 v_{F} p_{F}$ is an energy scale of the order of the Fermi energy, and $N_{F} \simeq p_{F}^{2} /\left(2 \pi^{2} v_{F}\right)$ is the density of states per spin at the Fermi level. Notice that the transverse spin-wave only emerges for $q \ll \delta$ with a dispersion $\omega_{s}(q) \simeq v_{F} \delta\left(q / 2 p_{F}\right)^{2}$.

On the paramagnetic (PM) side, the spin rotation symmetry is restored. The spin fluctuations become isotropic, $D_{\perp}=D_{\|} \equiv D$. All three modes take on the paramagnon form in Eqs. (11) with $\alpha$ determined by the spin correlation length $\xi_{s}, \alpha \sim \xi_{s}^{-2}$.

FM spin fluctuations are known to be pair-breaking 
in the s-wave channel and therefore suppress the conventional phonon-mediated superconductivity in PM metals. This problem was studied by Berk and Schrieffer [16] using the strong coupling Eliashberg theory and is believed to be the reason for why transition metals close to the FM instability have, if any, a low $T_{c}$. In contrast, the pairing interaction is attractive in the $l=$ odd angular momentum channel, raising the interesting possibility of FM spin fluctuation mediated spin-triplet superconductivity 17]. In general, the presence of a spontaneous magnetization in the FM phase leads to a set of four Eliashberg equations. However, for weak ferromagnets with small moments, the spin dependence of the self energy and the gap function can be ignored. For notational convenience, we shall limit the presentation to the PM phase, the modification on the FM side is straightforward and the differences will be noted explicitly.

Expressing the self energy in the Nambu formalism, $\Sigma_{p}(\omega) \equiv\left[1-Z_{p}(\omega)\right] \omega \tau_{0}+Z_{p}(\omega) \Delta_{p}(\omega) \tau_{1}$, where $\Delta_{p}(\omega)$ is the gap function, the linearized Eliashberg equations for the self-energy and the gap function are given by [18],

$$
\begin{aligned}
i \omega_{n}\left[1-Z_{p}\left(i \omega_{n}\right)\right]= & -g_{0}^{2} s_{0} T \sum_{i \epsilon_{n}} \int \frac{d^{3} k}{(2 \pi)^{3}} \frac{1}{i \epsilon_{n} Z_{k}\left(i \epsilon_{n}\right)-\xi_{k}} \\
& \times D\left(\vec{p}-\vec{k}, i \omega_{n}-i \epsilon_{n}\right), \\
Z_{p}\left(i \omega_{n}\right) \Delta_{p}\left(i \omega_{n}\right)= & g_{0}^{2} s_{l} T \sum_{i \epsilon_{n}} \int \frac{d^{3} k}{(2 \pi)^{3}} \frac{Z_{k}\left(i \epsilon_{n}\right) \Delta_{k}\left(i \epsilon_{n}\right)}{\epsilon_{n}^{2} Z_{k}^{2}\left(i \epsilon_{n}\right)+\xi_{k}^{2}}
\end{aligned}
$$

$$
\times D\left(\vec{p}-\vec{k}, i \omega_{n}-i \epsilon_{n}\right) .
$$

Here, $g_{0}$ is the coupling constant of the quasiparticles to spin fluctuations and $\xi_{k}=k^{2} / 2 m-\mu$. Note that the presence of Heisenberg symmetry in the PM phase guarantees three identical soft modes contributing to the self-energy but one longitudinal mode to the gap equation for triplet (p-wave) pairing [6], i.e. $s_{0}=3$ and $s_{1}=1$. To study the influence of the departure from this symmetry on $T_{c}$, such as in the case of Ising spins with $s_{0}=s_{1}=1$, we keep $s_{0}$ and $s_{l}$ as general parameters [8]. On the FM side, spin rotation symmetry is broken. While the gap equation (4) only contains the longitudinal mode and stays invariant, the self-energy equation (3) needs to be modified in a straightforward manner to reflect the different contributions from the transverse modes.

It is customary to proceed by separating the $k$ integral into angular and amplitude parts, $\int d^{3} k \rightarrow$ $\left(4 p_{F}^{2} / v_{F}\right) \int x d x \int d \phi \int d \xi_{k}$, where $x=\sin (\theta / 2), \theta$ is the angle between $\vec{k}$ and $\vec{q}=\vec{p}-\vec{k}$, and $\xi_{k}$ satisfies the kinematic constraint $q^{2} \simeq 4 p_{F}^{2} x^{2}+\xi_{k}^{2} / v_{F}^{2}$. As it will turn out later, close to the $\mathrm{QCP}$ and compared to their frequency dependence, the self energies have a negligibly weak momentum dependence. We thus drop the $k$-dependence in $Z_{k}(i \omega) \rightarrow Z(i \omega)$ and project the gap function into the $l$ th angular momentum channel, $\Delta_{k}(i \omega) \rightarrow \Delta_{l}(i \omega)$. With this approximation, it is possible to carry out the integral over $\xi_{k}$. In order to make analytical progress, we analytically continue to real frequencies and obtain,

$$
\begin{aligned}
{[1-Z(\omega)] \omega=-s_{0} g^{2} \int_{-\infty}^{\infty} d \epsilon \int_{0}^{1} x d x\left[\left(\frac{1}{U(\omega-\epsilon)[U(\omega-\epsilon)-i \epsilon Z(\epsilon) / \Lambda]}+\left(i Z \rightarrow-i Z^{*}\right)\right) \tanh \left(\frac{\epsilon}{2 T}\right)\right.} \\
\left.+\left(\frac{1}{U(\epsilon)[U(\epsilon)-i(\omega-\epsilon) Z(\omega-\epsilon) / \Lambda]}-\left(U \rightarrow U^{*}\right)\right) \operatorname{coth}\left(\frac{\epsilon}{2 T}\right)\right], \\
Z(\omega) \Delta_{l}(\omega)=s_{l} g^{2} \int_{-\infty}^{\infty} d \epsilon \int_{0}^{1} x d x\left[\left(\frac{\Delta_{l}(\epsilon)}{\epsilon} \frac{1}{U(\omega-\epsilon)[U(\omega-\epsilon)-i \epsilon Z(\epsilon) / \Lambda]}+\left(i Z, \Delta_{l} \rightarrow-i Z^{*}, \Delta_{l}^{*}\right)\right) \tanh \left(\frac{\epsilon}{2 T}\right)\right. \\
\left.+\left(\frac{\Delta_{l}(\omega-\epsilon)}{\omega-\epsilon} \frac{1}{U(\epsilon)[U(\epsilon)-i(\omega-\epsilon) Z(\omega-\epsilon) / \Lambda]}-\left(U \rightarrow U^{*}\right)\right) \operatorname{coth}\left(\frac{\epsilon}{2 T}\right)\right] P_{l}\left(1-2 x^{2}\right),
\end{aligned}
$$

where $g^{2}=g_{0}^{2} N_{F}^{2} / 2, P_{l}(x)$ is the Legendre polynomial, and $U^{2}(\epsilon)=\alpha+x^{2}-i \pi \epsilon / 4 x \Lambda$.

We first solve Eq. (5) to derive the quasiparticle self energy in the normal state. The self consistency in the this equation is crucial near the QCP, since as $\alpha \rightarrow 0$, the important low frequency cutoff in the denominators is the self energy itself. Carrying out the integrals, it is straightforward to show that the dominant contributions to the self energy come from the scattering by the spin fluctuations with momentum transfer $q \gg \delta$ and having the same form in both the PM and the FM phase close to the QCP. We find that the characteristic energy scale for spin fluctuation, $T^{*} \sim \alpha^{3 / 2} \Lambda$, enters as an important crossover temperature scale. For $y=\max (T, \epsilon)<T^{*}$, the self energy behaves as in a Fermi liquid,

$$
\Sigma(\epsilon, T) \approx-c^{\prime} \epsilon \ln \left(\Lambda / T^{*}\right)-i c^{\prime \prime} y^{2} / T^{*}, \quad y<T^{*},
$$

where $c^{\prime}, c^{\prime \prime} \sim s_{0} g^{2}$. However, for $y>T^{*}$, the scattering by spin fluctuations is enhanced and the self energy becomes non Fermi liquid like with the real part,

$$
\Sigma^{\prime}(\epsilon, T) \approx-c^{\prime} \epsilon \ln (\Lambda / y), \quad y>T^{*} .
$$

This leads to a quasiparticle residue $\mathcal{Z}, \mathcal{Z}^{-1}=1-$ $\left.\left[\partial \Sigma^{\prime} / \partial \epsilon\right]\right|_{\epsilon=0}=1+c^{\prime} \ln \left[\Lambda / \operatorname{Max}\left(T^{*}, T\right)\right]$, that vanishes logarithmically on approaching the QCP as in a marginal Fermi liquid [19]. For $\epsilon>T \gg T^{*}$, the imaginary part of $\Sigma$ follows, 


$$
\Sigma^{\prime \prime}(\epsilon) \approx-c^{\prime \prime} \pi \epsilon / 2, \quad \epsilon>T \gg T^{*} .
$$

In the quasistatic regime, $T>\epsilon \gg T^{*}$, another energy scale arises by comparing the coherence length $\xi_{\text {coh }} \sim$ $v_{F} / T$ to the spin correlation length $\xi_{s} \sim 1 / \sqrt{\alpha}$. We find for $\xi_{\mathrm{coh}}>\xi_{s}$,

$$
\Sigma^{\prime \prime}(T) \approx-c^{\prime \prime} T \ln \frac{T}{T^{*}}, \quad \frac{T}{\Lambda} \ln \frac{\Lambda}{T} \ll\left(\frac{T^{*}}{\Lambda}\right)^{1 / 3} .
$$

For even smaller $T^{*}$, such that $\xi_{\mathrm{coh}}<\xi_{s}$, the quasiparticles scatter off essentially uncorrelated spins. In this case, the unphysical singularity in Eq. (10) as $T^{*} \rightarrow 0$ must be removed by the self-consistency of Eq. (5) where $\Sigma^{\prime \prime}$ itself becomes the cutoff. We find

$$
\Sigma^{\prime \prime} \approx-c^{\prime \prime} T \ln \left(\Lambda^{2} T /\left|\Sigma^{\prime \prime}\right|^{3}\right)+3 c^{\prime \prime} \sqrt{\alpha} T \Lambda / 2\left|\Sigma^{\prime \prime}\right|,
$$

which has the following self-consistent solution,

$$
\Sigma^{\prime \prime} \approx-c^{\prime \prime} T \ln \frac{\Lambda}{T}+\frac{3}{2} c^{\prime \prime} \sqrt{\alpha} \Lambda, \quad \frac{T}{\Lambda} \ln \frac{\Lambda}{T} \gg\left(\frac{T^{*}}{\Lambda}\right)^{1 / 3} .
$$

The same behavior of $\Sigma$ holds on the FM side since the dominant scattering process has $q \gg \delta$. In this range of $q$, the breaking of spin rotation invariance is insignificant as seen in Eqs. (11) and (2). The spin wave contribution is not important since the difference between the Fermi momentum $(\delta)$ is much larger than most of the momenta carried by the spin waves. Notice that the inelastic scattering rate in quasistatic limit increases with a square-root singularity as $\alpha \rightarrow 0$, leading eminently to a rapid suppression of the superconducting transition temperature $T_{c}$ on approaching the QCP.

We next determine $T_{c}$ by solving Eqs. (6) for the simplest $l=1$, p-wave case 20 To treat the effects of both mass renormalization and scattering lifetime, we write $Z=Z^{\prime}+i Z^{\prime \prime}$ and the complex gap function as $\Delta=\Delta^{\prime}+i \Delta^{\prime \prime}$. Taking the imaginary part of the gap equation (6), we obtain to leading order in $T_{c} / T_{0}, T_{0} \sim \Lambda$ being the cutoff frequency for spin fluctuations - a magnetic analog of Debye frequency,

$$
Z^{\prime}(\omega) \Delta^{\prime \prime}(\omega)+\beta Z^{\prime \prime}(\omega) \Delta^{\prime}(\omega) s_{l} / s_{0} \simeq 0 .
$$

Here $\beta=s_{0} / s_{l}-1$ reflects the spin symmetry. Writing for small $\omega, \omega Z(\omega)=[1+\lambda(T)] \omega-i \Gamma(\omega)$, where $\lambda=-\Sigma^{\prime} / \omega$ is the effective coupling and $\Gamma=-\Sigma^{\prime \prime}$ is half the inverse life-time of the quasiparticles, Eq. (13) becomes $\beta \Gamma(\omega) \Delta^{\prime}(\omega)=(1+\lambda) \omega \Delta^{\prime \prime} s_{0} / s_{l}$, which allows us to account for the damping of the order parameter in terms of a real effective gap function,

$$
\Delta_{\mathrm{eff}}(\omega)=\Delta^{\prime}(\omega)\left[1+(\widetilde{\Gamma} / \omega)^{2}\right],
$$

where $\widetilde{\Gamma}=\beta s_{l} \Gamma / s_{0}(1+\lambda)$. In contrast to $\Delta^{\prime}, \Delta_{\text {eff }}$ has a weaker $\omega$-dependence and remains finite in the small $\omega$ limit. Now we can rewrite the real part of the gap equation (6) as an integral equation for $\Delta_{\text {eff }}$,

$$
\begin{gathered}
(1+\lambda) \Delta_{\mathrm{eff}}(\omega)=2 s_{l} g^{2} \int_{-\infty}^{\infty} d \epsilon \int_{0}^{1} x d x P_{l}\left(1-2 x^{2}\right) \\
\tanh \left(\frac{\epsilon}{2 T_{c}}\right) \frac{\epsilon \Delta_{\mathrm{eff}}(\epsilon)}{\epsilon^{2}+\widetilde{\Gamma}^{2}\left(\epsilon, T_{c}\right)} \operatorname{Re} \frac{1}{U^{2}(\omega-\epsilon, x)}
\end{gathered}
$$

The scattering rate enters as a low-energy cutoff of the logarithmic singularity responsible for the superconducting instability. Next we attempt an approximate analytical solution of $T_{c}$ from Eq. (15) where the weak frequency dependence of $\Delta_{\text {eff }}$ can be neglected.

Fermi liquid regime. Consider first the case $T_{c} \ll$ $T^{*} \ll T_{0}$, i.e. the onset of superconductivity in the Fermi liquid regime away from the QCP. Since the temperature is much lower than the characteristic spin fluctuation frequency, inelastic scattering dominates, but with the ordinary Fermi liquid scattering rate (see Eq. 7) that is much smaller than $\max \left(k_{B} T, \epsilon\right)$. The effect of a nonzero $\widetilde{\Gamma}$ on $T_{c}$ is thus small and negligible. Solving Eq. (15) to next to leading order in $T^{*} / T_{0}$, we obtain

$$
T_{c} \simeq T_{0} e^{-\left[\eta^{\prime}+\beta(2 A+3)+A^{2}\right] /(2 A-3)},
$$

where $A=\ln T_{0} / T^{*}, \quad \eta^{\prime}=\left(3 / 2 s_{l} g^{2}\right)+\left(\pi^{2} / 24\right)+$ $6 \ln (\sqrt{2} \gamma / \pi)-3$, and $\ln \gamma \simeq 0.577$ is Euler's constant. As $T^{*}$ is reduced towards the QCP, spin fluctuations increase and pairing is enhanced. This causes $T_{c}$ to rise initially. Reducing $T^{*}$ further eventually causes the system near $T_{c}$ to lose sensitivity to the finite correlation length, leading to new physics associated with superconductivity near quantum criticality.

Quantum critical region. Here, $T_{0} \gg T_{c} \gg T^{*}$, the superconducting transition occurs inside the quantum critical regime. Since the temperature is much higher than the characteristic quantum spin fluctuation energy, inelastic scattering is negligible and the dominant pairbreaking effect comes from quasistatic $\left(\omega<T_{c}\right)$ spin fluctuations with a scattering rate $\widetilde{\Gamma}(T)=-\beta s_{l} \Sigma^{\prime \prime}(T) / s_{0}[1+$ $\lambda(T)]$. The suppression of $T_{c}$ due to $\widetilde{\Gamma}$ is thus reminiscent of Abrikosov and Gor'kov's theory of superconducting alloys with paramagnetic impurities 14. Accordingly, Eq. (15) has the solution,

$$
\ln \frac{T_{c}}{T_{c 0}}=\psi\left(\frac{1}{2}\right)-\psi\left[\frac{1}{2}+\frac{\widetilde{\Gamma}\left(T_{c}\right)}{2 \pi T_{c}}\right],
$$

where $\psi$ is the digamma function and $T_{c 0}$ is the transition temperature in the absence of $\widetilde{\Gamma}$. To leading order in $T c / T_{0}$,

$$
\frac{T_{c 0}}{T_{0}}=e^{-\left[\tilde{\beta}+\sqrt{\tilde{\beta}^{2}+2 \beta\left[1-\frac{\pi}{\sqrt{3}}\left(\frac{T^{*}}{T_{c 0}}\right)^{2 / 3}\right]+\eta+\frac{\pi}{\sqrt{3}}\left(\frac{T^{*}}{T_{c 0}}\right)^{2 / 3}}\right.},
$$

with $\tilde{\beta}=3+\beta$ and $\eta=\left(3 / 2 s_{l} g^{2}\right)+6 \ln (2 \gamma / \pi)-\pi^{2} / 24$. At the QCP, we find $T_{c 0} / T_{0} \sim 10^{-5}$ for Heisenberg symmetry and $\sim 10^{-3}$ for Ising symmetry. 
A few remarks are in order for $T_{c}$ in the quantum critical regime. (i) For Ising spins, $\beta=0$. Eq. (17) shows that $T_{c}$ is not affected by a finite quasiparticle life-time, i.e. $T_{c} \simeq T_{c 0}$. Furthermore, to leading order, $T_{c 0}$ is not reduced by the real part of the self energy. This is in fact a manifestation of Anderson's theorem [21] for nonmagnetic impurities. It arises in our case from the cancellation of the self-energy effects in the gap equation (15) in the quasistatic limit when $s_{0}=s_{l}$. From Eq. (18), it follows that $T_{c}$ decreases linearly with $\alpha$ close to the QCP with a slope $d T_{c} / d \alpha \sim-T_{c}\left(T_{0} / T_{c}\right)^{2 / 3}$ for Ising spins.

(ii) For Heisenberg spins, $\beta=2 . T_{c 0}$ decreases with the reduction of quasiparticle coherence on approaching the QCP. $T_{c}$ is further reduced from $T_{c 0}$ due to the increasing scattering rate. However, in contrast to the case of magnetic impurities where $T_{c}$ can be suppressed to zero at a finite concentration, we find that $T_{c}$ remains finite at the $Q C P$ as a consequence of the $T$-dependent scattering rate. From Eqs. (8.12) at $\alpha=0$, it follows that $\widetilde{\Gamma}\left(T_{c}\right) / T_{c}=\beta s_{l} c^{\prime \prime} \ln \left(\Lambda / T_{c}\right) / s_{0}\left[1+c^{\prime} \ln \left(\Lambda / T_{c}\right)\right]$ tends to a constant $\rho=c^{\prime \prime} \beta s_{l} / 2 s_{0} \pi c^{\prime}$ of order unity for $T_{c} \ll \Lambda$, leading to $T_{c} \simeq T_{c 0} \exp -[\psi(1 / 2+\rho)-\psi(1 / 2)]$.

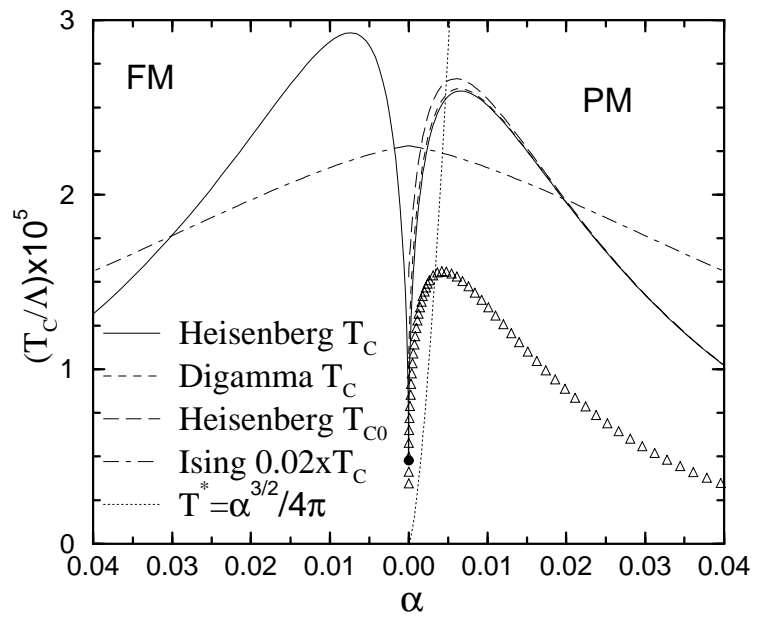

FIG. 1. $\quad T_{c}$ versus $\alpha$ for $g^{2}=0.3$ (lines) and 0.15 (triangles). $T_{c 0}$ and $T_{c}$ obtained from Eq. (17) are shown for comparison. The peak in $T_{c}$ versus $\alpha$ scales with $T^{*}$.

The results obtained from numerical solution of Eq. (15) are shown in Fig. 1. On the PM side, they are in agreement with those of Roussev and Millis [8]. We next analyze how $T_{c}$ varies with $\alpha$ close to the QCP in the Heisenberg case. Eq. (18) shows that $T_{c 0}$ increases linearly with $\alpha$, as the overall sign of the terms proportional to $\left(T^{*} / T_{c}\right)^{2 / 3}$ has changed from the Ising case due to the real part of the self energy when $\beta=2$. However, we find that this effect is subleading, and the dominant $\alpha$-dependence of $T_{c}$ comes from the scattering rate in Eq. (17) through the strong $\alpha$-dependence of $\Sigma^{\prime \prime}$ near the QCP, i.e. $d T_{c} / d \alpha \propto-d \Sigma^{\prime \prime} / d \alpha$. From Eqs. (10, 12), we obtain, $d T_{c} / d \alpha \sim 1 / \sqrt{\alpha}$, for $\sqrt{\alpha} \ll\left(T_{c} / \Lambda\right) \ln \left(\Lambda / T_{c}\right)$; and $d T_{c} / d \alpha \sim 1 / \alpha$, for $\left(T_{c} / \Lambda\right) \ln \left(\Lambda / T_{c}\right)<\sqrt{\alpha}<\left(T_{c} / \Lambda\right)^{1 / 3}$.

In the FM phase, the spin rotation symmetry is broken. However, close to the transition, approximate Heisenberg symmetry is restored due to the small difference in the Fermi momenta which in turn, as discussed above, suppresses the contributions of the long wavelength Goldstone mode to the electron-spin fluctuation kernel. As a result, the superconducting phase boundary is approximately symmetric near the magnetic QCP. Away from the QCP, the deviation from the Heisenberg symmetry leads effectively to a $\beta$ value that is shifted downward from the Heisenberg value and a somewhat higher $T_{c}$ (see Fig. 1) on the FM side. Well inside the FM phase, the relative suppression of the fluctuation in the transverse channel makes the situation closer to the Ising case studied above, resulting in a much higher $T_{c}$ [2].

To conclude, our results suggest whether a significant suppression of $T_{c}$ occurs near the QPC can be used to help identify the spin symmetry of the superconducting order parameter. We have shown that such a reduction occurs in the triplet case but is absent for singlet pairing, e.g. the s-wave pairing proposed in the weak ferromagnetic local Fermi liquid theory [9]. In the singlet case, the spin fluctuations contributing to the self energy and the gap equation are identical and the dominant quasistatic pair-breaking effects in the quantum critical regime cancel out as in the Ising case discussed above. While more experiments are clearly needed to determine the phase diagram near the FM QCP [2], existing data in the AF case [1] show that $T_{c}$ indeed peaks near the magnetic QCP where pairing due to AF spin fluctuations is expected to be of spin-singlet in nature.

This work is supported in part by DOE Grant Nos. DE-FG02-99ER45747, DE-FG02-97Er45636, and by an award from Research Corporation.

[1] N. D. Mathur, et. al. Nature 394, 39 (1998).

[2] S. S. Saxena, et. al. Nature 406, 587 (2000).

[3] Z. Fisk and D. Pines, Nature 394, 22 (1998)

[4] P. Coleman, Nature 406, 508 (2000).

[5] I. I. Mazin and D. J. Singh, Phys. Rev. Lett. 78, 3374 (1997).

[6] P. Monthoux and G. G. Lonzarich, Phys. Rev. B59, 14598 (1999).

[7] A. Abanov, et. al., cond-mat/0005163.

[8] R. Roussev and A. J. Millis, Phys. Rev B63, 140504 (2001).

[9] K. B. Blagoev, et. al. Phys. Rev. Lett. 82, 133 (1999).

[10] C. Pfleiderer, et. al. Phys. Rev. B55, 8330 (1997).

[11] I.E. Dzyaloshinskii and P.S. Kondratonko, Sov. Phys. JETP, Vol. 43, No.5, 1036(1976).

[12] S. Doniach and S. Engelsberg, Phys. Rev. Lett. 17, 750 (1966).

[13] A. J. Millis, Phys. Rev. B48, 7183 (1993); J. Hertz, Phys. Rev. B14, 1165 (1976). 
[14] A. A. Abrikosov and L. P. Gor'kov, Sov. Phys. JETP 12, 1243 (1961).

[15] E. I. Blount and C. M Varma, Phys. Rev. Lett. 42, 1079 (1979); T. K. Ng and C. M. Varma, Phys. Rev. B58, 11624 (1998).

[16] N. F. Berk and J. R. Schrieffer, Phys. Rev. Lett. 17, 433(1966).

[17] D. Fay and J. Appel, Phys. Rev. B22, 3173 (1980).
[18] D. J. Scalapino, in Superconductivity, edited by R. D. Parks (Marcel Dekker, New York) 1969.

[19] C. M. Varma, et. al. Phys. Rev. Lett. 63, 1996 (1989).

[20] Since the pairing interaction near the QCP is isotropic to leading order in the magnetization, the three components of the p-wave order parameter are degenerate.

[21] P. W. Anderson, J. Phys. Chem. Solids 11, 26 (1959). 